\title{
Hypoplastic left heart syndrome with an intact atrial septum
}

\author{
Vikram Sood, MD, ${ }^{\mathrm{a}}$ Jeffrey D. Zampi, MD, ${ }^{\mathrm{b}}$ and \\ Jennifer C. Romano, MD, MS ${ }^{\mathrm{a}}$
}

\begin{abstract}
Feature Editor's Note-Newborns with hypoplastic left ventricle and intact atrial septum are among the most challenging subgroups of patients. Despite the fact that most patients are now diagnosed antenatally by fetal echocardiography, the mortality in these patients remains high and the long-term prognosis is dismal. Herein, $\mathrm{Dr}$ Romano and colleagues present a team-based hybrid approach developed by the University of Michigan multidisciplinary group. One can only hope that this admirable, labor-intense collaborative effort by fetal and interventional cardiologists, pediatric cardiothoracic surgeons, pediatric intensivists, and palliative care and social work specialists will overcome the severity of the underlying condition and improve the outcomes of these patients.
\end{abstract}

\section{Igor E. Konstantinov, MD, PhD, FRACS}

Despite improving outcomes in staged, single-ventricle palliation for hypoplastic left heart syndrome (HLHS), the rare (approximately $6 \%$ of all HLHS) subgroup of HLHS with an intact (or highly restrictive) atrial septum (IAS) continues to have mortality rates in excess of $50 \%$. ${ }^{1}$ The Children's Hospital of Philadelphia group published sobering results in 2007, highlighting the lethality of HLHS/IAS with 30-day survival of $38 \%$ despite emergent postnatal intervention. $^{2}$

At birth, in the setting of HLHS, there is obligate left to right flow across a patent foramen ovale (or secundum atrial septal defect) that allows oxygenated pulmonary venous return to get to the right side of the heart to be ejected to the body. In HLHS/IAS, there is an inability of oxygenated

\footnotetext{
From the ${ }^{\text {a Section }}$ of Pediatric Cardiac Surgery, Department of Cardiac Surgery, and ${ }^{\mathrm{b}}$ Section of Pediatric Cardiology, Department of Pediatrics, University of Michigan Health Systems, Ann Arbor, Mich.

Received for publication Feb 18, 2020; revisions received Feb 18, 2020; accepted for publication March 11, 2020.

Address for reprints: Jennifer C. Romano, MD, MS, 1540 E Hopsital Dr, SPC 4204, Section of Pediatric Cardiac Surgery, Department of Cardiac Surgery, University of Michigan Health Systems, C.S. Mott Children's Hospital, Ann Arbor, MI 481094240 (E-mail: jhirsch@umich.edu).

JTCVS Open 2020;1:51-6

2666-2736

Copyright (ㄷ 2020 by The Authors. Published by Elsevier Inc. on behalf of The American Association for Thoracic Surgery. This is an open access article under the CC BY-NC-ND license (http://creativecommons.org/licenses/by-nc-nd/4.0/).

https://doi.org/10.1016/j.xjon.2020.03.001
}

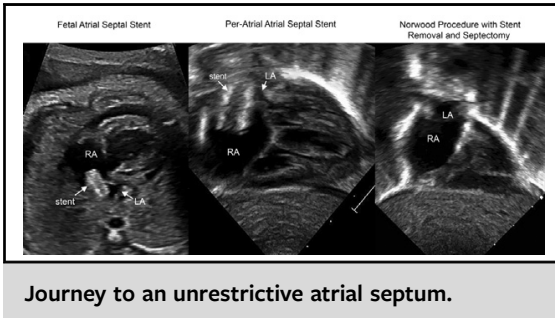

CENTRAL MESSAGE

Our team-based hybrid

approach was developed to improve early stability and, we hope, long-term outcomes in the rare but high-risk lesion called hypoplastic left heart syndrome with intact atrial septum.

See Commentaries on pages 57 and 59 .

blood to get to the right side of the heart due to the intact or highly restrictive atrial septum. This results in profound systemic hypoxemia. In addition, left atrial hypertension leads to pulmonary edema and elevated resistance to antegrade pulmonary blood flow that further exacerbates the hypoxemia.

The theorized etiology for the high mortality with HLHS/ IAS centers on the pulmonary vascular changes during gestation as a result of a hypertensive left atrium. These neonates, even after undergoing relief of atrial level obstruction (ie, stage 1 palliation) continue to demonstrate elevated pulmonary vascular resistance (PVR). ${ }^{3}$ Histopathology of lung biopsies from these patients has shown marked lymphangiectasia and pulmonary hypoplasia that is suggestive of end-stage lung injury. ${ }^{1,4}$ As such, attention has been turned toward in utero intervention to prevent pulmonary vascular changes and lung injury."

Fetal balloon atrial septostomy and septal stenting have been utilized to improve development of the pulmonary vasculature and prevent left atrial hypertension. Fetal intervention has previously been described with a maternal, transabdominal approach and a fetal, transthoracic puncture, utilizing atrial septal balloon septostomy and stent placement. Fetal intervention has been shown to improve postnatal hemodynamic stability; however, most neonates 


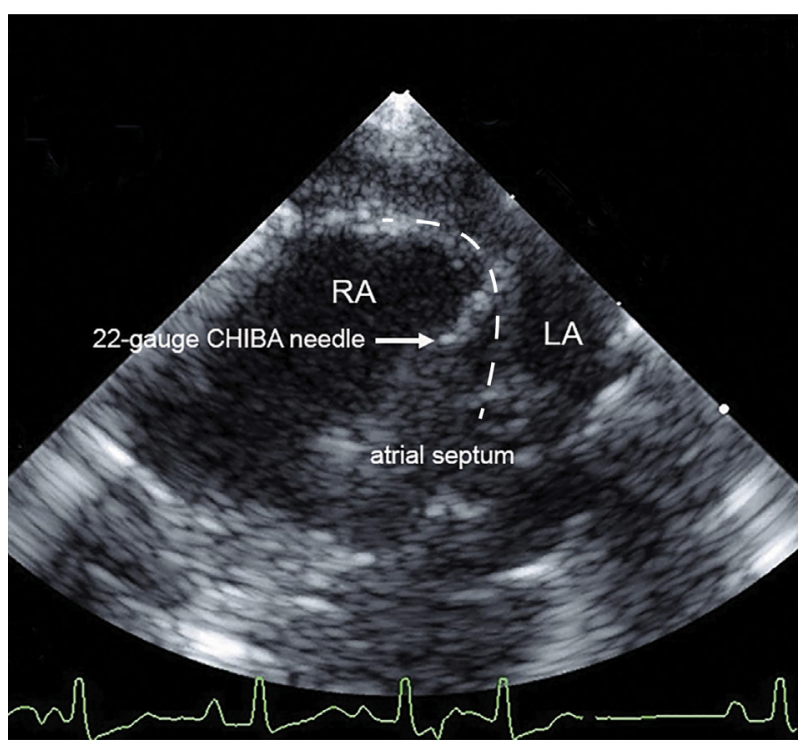

FIGURE 1. Transesophageal echocardiogram assisted transseptal access with a 22-gauge CHIBA needle (Cook Medical, Bloomington, Ind). $R A$, Right atrium; $L A$, left atrium.

still require early postnatal intervention to durably relieve atrial septal obstruction and hypoxemia. ${ }^{6}$ The Boston group has published results that demonstrate improving early survival in patients with HLHS/IAS treated with fetal intervention compared with those who were treated only postnatally. ${ }^{7}$ Fetal intervention is limited by the timing of diagnosis, fetal position, and ability to create a nonrestrictive interatrial communication. As such, many patients with HLHS/IAS are not candidates for this potentially beneficial strategy.

Neonates with HLHS/IAS without fetal intervention require urgent postnatal intervention to relieve the septal obstruction and improve oxygenation. ${ }^{8,9}$ Even with a decompressing levoatrial vein or a prior fetal intervention, many will require urgent postnatal intervention. In the current era, most single-ventricle patients are identified on fetal echocardiogram. In patients with HLHS/IAS or highly restrictive atrial septum, a postnatal intervention-percutaneous balloon septostomy, surgical septostomy, or peratrial septal balloon septostomy/stenting - is typically arranged to rapidly follow delivery.

\section{UNIVERSITY OF MICHIGAN STRATEGY}

Our approach to HLHS/IAS is team-based and utilizes the expertise of many different provider-groups to obtain the best likelihood of success in this challenging patient population as well as to support the family in decision making. When a prenatal diagnosis is made, we initiate a collaborative approach, including fetal cardiology, interventional cardiology, pediatric cardiac surgery, palliative care, and social work. It is essential to develop a unified plan to support the family and the unique needs of each fetus. First, we assess for the feasibility of fetal intervention. Our preference is atrial septal stenting rather than balloon dilation to provide a durable atrial septal defect until the time of delivery. Gestational age at diagnosis and fetal position are major determinants to feasibility. Regardless of whether a fetal intervention is performed, we recommend all mothers with a fetal diagnosis of HLHS/IAS undergo scheduled caesarean-section delivery for emergent postnatal intervention. Maternal parity and other factors, including noncardiac anomalies are weighed in this decision because future child-bearing interest needs to be considered. We do not offer urgent hybrid intervention in patients with severe prematurity/intrauterine growth restriction (IUGR), other major noncardiac anomalies/syndromes, or immediate postnatal arrest. In our experience, no referred patient has selected comfort care and 2 patients were not offered a postnatal intervention due to social issues and extreme IUGR.

In our institution, the women's hospital is within the children's hospital, allowing for the care and delivery of the mother to occur in close proximity to the care needs for the baby. Following elective caesarean-section delivery, rapid intubation, and umbilical venous and arterial line placement is performed. Intravenous prostaglandins are initiated to maintain ductal patency. A limited transthoracic echocardiogram is performed to confirm the diagnosis of HLHS/IAS and to assess the feasibility of ductal stenting. The patient is then expeditiously (within 30 minutes of delivery) transferred to our interventional cardiac catheterization suite. Our maternal-fetal medicine and neonatology colleagues have become adept at swiftly and safely delivering, triaging, providing a definitive airway and access, and transferring the neonate.

Once in the interventional suite, a transesophageal echocardiography (TEE) probe is placed. The patient is rapidly prepped and draped for sternotomy and peratrial balloon septostomy and/or atrial septal stenting. The goal is $<5$ minutes from arrival to peratrial access of the atrial septum. Rapid relief of the atrial-level obstruction is essential to relieve the profound hypoxemia and rapidly progressive acidosis. As such, the hybrid team, consisting of echocardiography, interventional cardiology, pediatric cardiac surgery, cardiac anesthesia, and operating room/interventional suite staff is fully set up before delivery and is scrubbed before the patient's transport.

After sternotomy, septal intervention is performed with echocardiography-guided placement of a $6 \mathrm{~F}$ sheath into the right atrium and perforation of the central portion of the septum with a 22-gauge CHIBA needle (Cook Medical, Bloomington, Ind) (Figure 1). The remainder of the procedure is performed using a combination of TEE and dual plane fluoroscopy. Following septal access, a guide wire is 


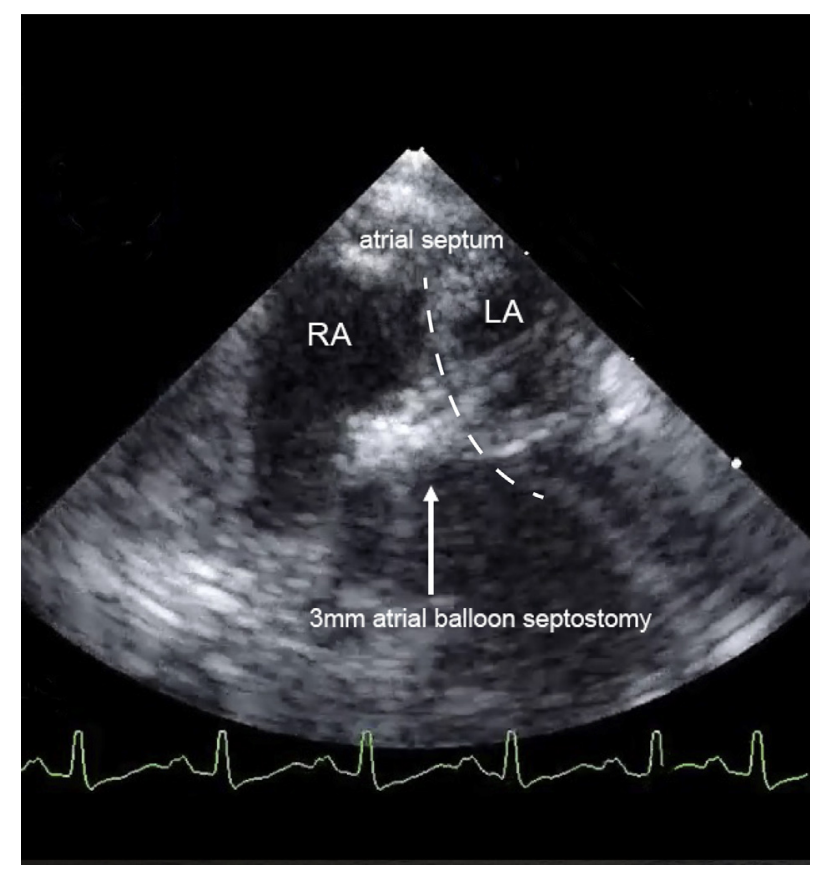

FIGURE 2. A 3-mm balloon is used to dilate the atrial septostomy. $R A$, Right atrium; $L A$, left atrium.

preferably placed into the left lower pulmonary vein to provide an ideal angle and stable wire position. A 3-mm balloon is passed over the wire and inflated across the atrial septum (static balloon atrial septoplasty) to create a small atrial egress (Figure 2). This allows a better assessment of the thickness of the atrial septum, which is typically thin and bowing into the right atrium initially, and allows for easier passage of further balloons or stents. If the septum is thin and mobile, a balloon septostomy is preferred and the septum is serially dilated with cutting balloons (up to $7 \mathrm{~mm}$ ) and high pressure noncompliant angioplasty balloons (up to $10 \mathrm{~mm}$ balloon diameter if feasible) ( $3 \mathrm{~mm}$ Maverick2 PTCA balloon and $7 \mathrm{~mm}$ Flextome cutting balloon [Boston Scientific, Marlborough, Mass] and $10 \mathrm{~mm}$ Advance 18LP PTA balloon dilation catheter [Cook Medical]). The septum can be tented/dragged toward the left atrium by the balloon; therefore, appropriate position of the balloon across the septum, which is confirmed by TEE, is essential for adequate decompression. If the atrial septum is thick, we instead proceed with atrial septal stent placement. The stent can be advanced and placed directly after the $3 \mathrm{~mm}$ balloon dilation (Figure 3). Stent diameter and length is based on the total length of the septum and distance from the septum to the left atrial free wall. Typically, an $8 \mathrm{~mm} \times 12 \mathrm{~mm}$ or $16 \mathrm{~mm}$ stent is used. With regard to balloon dilation versus atrial septal stenting, our practice is to place a stent whenever it is believed that a stable position can be achieved. We believe

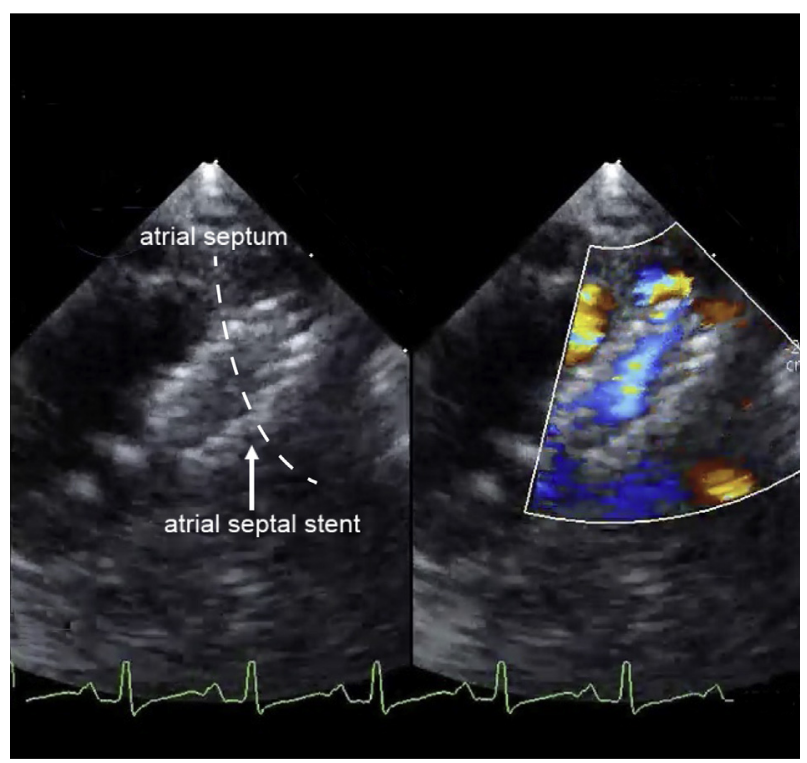

FIGURE 3. An atrial septal stent is positioned.

stenting to be the more durable methodology to achieve an un-restrictive atrial septum.

For patients who previously underwent fetal atrial septal stent placement, we typically perforate the atrial septum adjacent to the fetal stent and place a new stent using the same technique described above. Embolization of the fetal stent to the left atrium can occur but is typically of no clinical consequence and will be removed at the time of the eventual atrial septectomy.

After successful atrial septal intervention establishing unobstructed flow across the atrial septum, we proceed with bilateral pulmonary artery (PA) band placement and ductal stenting (Figure 4).

Pulmonary artery banding is challenging in this setting because the PVR may remain elevated due to the in utero atrial level obstruction. To guide adequate band placement, a RADI flow wire (RADI Medical Systems, Uppsala, Sweden) is used to assess pressure distal to the bands. ${ }^{12}$ This allows the bands to be placed with relatively equal distal PA pressures. Echocardiography is unreliable because of the equalization of band gradients that can lead to asymmetric bands as well as low initial gradients in the setting of elevated PVR. Changes in oxygen saturation with banding are also unreliable due to pulmonary parenchymal injury. A 6F sheath is placed in the proximal main PA just beyond the sinotubular junction. Through this sheath, a 4F JB-1 catheter (our preferred catheter is the Terumo Glidecath Hydrophilic Coated Catheter [Terumo Medical Corporation, Somerset, NJ]) is advanced across the ductus arteriosus into the descending aorta. The RADI wire is advanced through the JB-1 until the distal end is free of the catheter and then is zeroed or normalized to the arterial wave form obtained from the JB- 1 catheter. The JB- 1 followed by the 


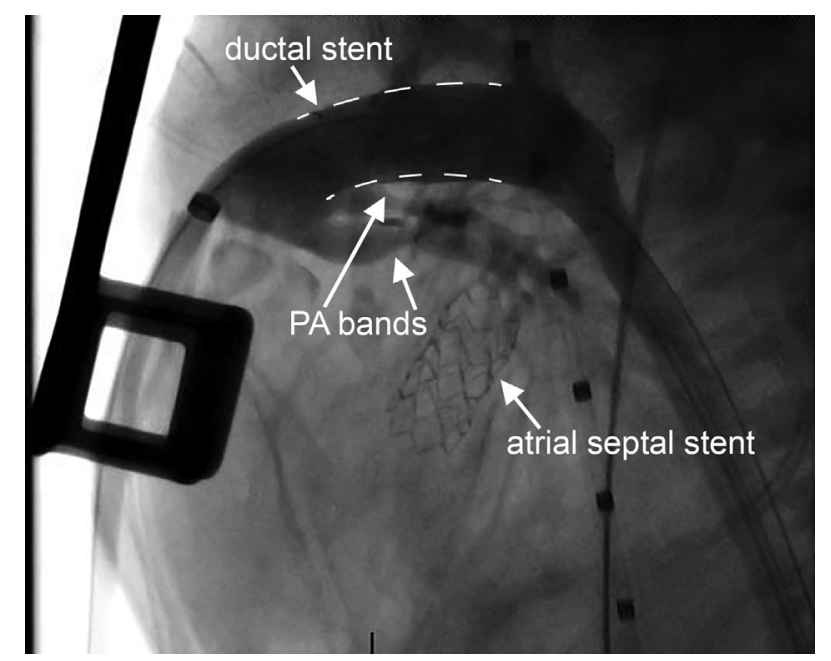

FIGURE 4. Fluoroscopy demonstrating urgent per-atrial septal stenting, bilateral pulmonary artery banding, and ductal stenting.

RADI wire is then advanced into the left PA. With the wire in place in the distal PA, it is essential to make sure the JB-1 is pulled back into the sheath so the catheter does not obstruct the PA orifice resulting in falsely low distal PA pressures and placement of a loose band. The PA bands are created by cutting 2-mm wide bands from either a $3.0 \mathrm{~mm}$ (patients $<3 \mathrm{~kg}$ ) or $3.5 \mathrm{~mm}$ (patients $>3 \mathrm{~kg}$ ) Gore-Tex tube graft (W.L. Gore and Associates, Newark, Del). With the RADI wire in place, the band is secured around the left PA with a horizontal Prolene (Ethicon, Bridgewater, NJ) mattress suture. Minimal dissection is used to gain circumferential control of the PA to reduce the potential for distal band migration. The band is adjusted as necessary with a single titanium clip to obtain a mean distal PA pressure of 15 to $20 \mathrm{~mm} \mathrm{Hg}$ with some pulsatility (3-5 $\mathrm{mm} \mathrm{Hg}$ ) of the distal PA pressure tracing. When $1 \mathrm{PA}$ is banded, the bulk of the blood flow is shunted to the contralateral PA due to reduced resistance within that vascular bed. Therefore, it can be anticipated that the left PA pressure will increase modestly once the right PA band is placed. Once the left PA band is adequate, the RADI wire is positioned in the right PA and the right band is placed in a similar fashion. Before completing this portion of the procedure, the left PA band is reassessed to confirm that it is appropriately restrictive. With HLHS/IAS, we anticipate most patients will require supplemental oxygen (up to $50 \%$ ) initially and final band determination is completely driven by the distal pressures and, to date, has been effective without need for readjustment. Transfusion with packed red blood cells is performed to ensure a hematocrit level above 40. We accept arterial oxygen tension above $30 \mathrm{~mm} \mathrm{Hg}$ and acute pulmonary vasodilator therapy with inhaled nitric oxide is rarely used. When the RADI wire was initially introduced into our hybrid stage 1 palliation (HS1P) program, the number of PA band adjustments during the procedure as well as additional interventions to assess and adjust bands was significantly reduced without any increase in the procedure or fluoroscopy time. ${ }^{10}$

After appropriately restricting pulmonary blood flow, ductal stenting is performed. Some centers prefer longterm prostaglandin E1 to maintain ductal patency after HS1P for HLHS. Our preference is to perform ductal stenting in the absence of prohibitive anatomy. In patients with aortic stenosis and evidence of reasonable antegrade flow, arch anatomy is not a factor and all undergo ductal stenting. With aortic atresia, if there is evidence of a so-called 3 sign with angulation of the transverse arch into the patent ductus arteriosus, ductal stenting is not performed, and patients are maintained on prostaglandin E1. In this scenario, patients are at risk for developing a reverse coarctation, where the ascending aorta and thus coronary arteries receive insufficient blood flow. Stenting allows for durable maintenance of ductal patency, minimizes the risk of long-term intravenous access, and allows for discharge home before the Norwood procedure, which is important for neonatal bonding and development. Although ductal stenting carries some risk, prostaglandin E1 can have significant side effects. In our recently published comparison of HS1P versus Norwood procedure in high-risk HLHS patients, $23 \%$ of hybrid patients did not undergo ductal stenting at the time of HS1P due to retrograde coarctation concerns. ${ }^{10}$

Postoperatively, all HS1P patients are managed in our dedicated pediatric cardiothoracic intensive care unit. Our aim in HLHS/IAS patients who have undergone HS1P is to perform a Norwood procedure at approximately 6 to 8 weeks of life. For premature infants, we wait until they have reached a corrected term gestational age and preferably a weight of 3.5 to $4 \mathrm{~kg}$ if born with IUGR. The delay is to allow normalization of PVR and healing of the fetal/ early neonatal lung injury. Discharge home before the surgical Norwood procedure even if for a short period of time is preferred. Weekly transthoracic echocardiograms while inpatient and biweekly once discharged to outpatient care are performed to assess the atrial septum, ductal patency/reverse coarctation, and ventricular/tricuspid valve function. In our experience, any change in systemic ventricular function or tricuspid regurgitation warrants vigilant assessment of reverse coarctation or ductal restriction. Further, recurrent atrial septal restriction should be addressed immediately and aggressively to prevent further worsening of lung injury and elevated PVR. Due to the complexity of these patients, we require ongoing, shared follow-up at our center and with the primary cardiologist during this phase.

During the Norwood procedure, the atrial septal stent(s) are removed and any residual atrial-level obstruction is addressed, the PA bands are removed and surrounding scar is released, and a standard Norwood is performed usually with 


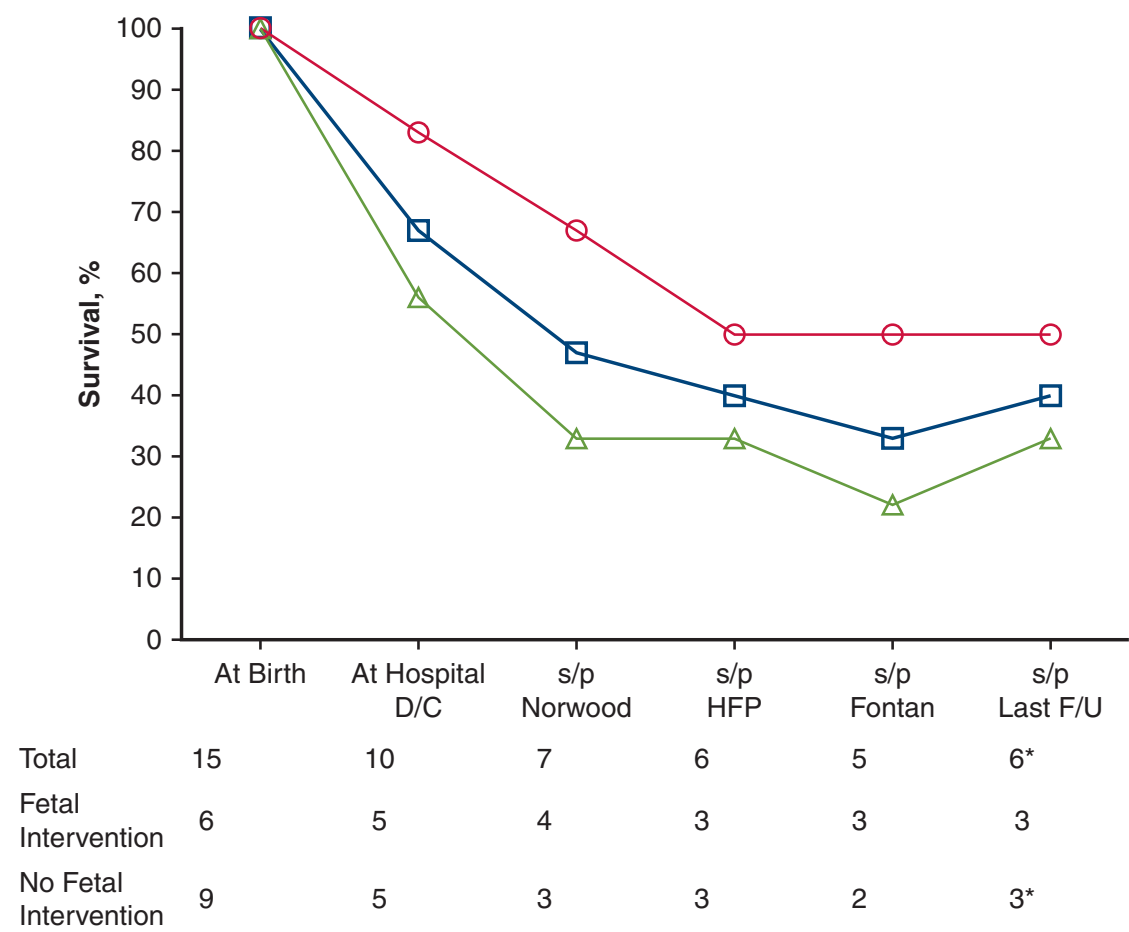

HLHS/IAS = hypoplastic left heart syndrome with intact atrial septum, $d / c=$ discharge, HFP $=$ hemi-Fontan procedure

* 1 Orthotopic heart transplant

$\boxminus$ Total $\ominus$ Fetal intervention $\triangle$ No Fetal intervention

FIGURE 5. Patient outcomes with our hybrid protocol for hypoplastic left heart syndrome with intact atrial septum. $D / C$, Discharge; $s / p$, status post; $H F P$, hemi-Fontan procedure; $F / U$, follow-up. *1 orthotopic heart transplant.

placement of a modified Blalock-Taussig shunt to encourage PA growth. The remainder of staged singleventricle palliation will then follow the typical time frame with hemi-Fontan at 4 to 6 months post-Norwood and lateral tunnel Fontan at age 2 to 3 years. Most patients will need to be maintained on sildenafil through all 3 stages to minimize PVR with hope for a long-term wean.

At the time of the HS1P, a representative biopsy from either lung is typically obtained in patients with HLHS/ IAS to delineate the degree of pulmonary hypoplasia and/ or angiocapillary dysplasia, which are common findings with this lesion. Although biopsy has not been used to drive clinical decision making, it often provides some helpful prognostic data to inform counseling of parents. It is hoped that over time with an increasing number of pathologic specimens, histopathologic findings can be correlated with the clinical course.

\section{DISCUSSION}

High rates of morbidity and mortality are associated with HLHS/IAS despite advances in overall survival of HLHS patients. Although fetal intervention has led to improved survival rates in select HLHS/IAS patients amenable to the therapy, many still require emergency postnatal intervention. To date, no patient undergoing our protocol for HLHS/IAS has had a reassuring atrial septal defect at birth. Moreover, even in those patients who are relatively more stable as a result of a decompressing levo-atrial vein, our institutional preference is to move forward with initial emergent HS1P.

The University of Michigan approach to high-risk, single-ventricle lesions such as HLHS/IAS is with a teambased HS1P. Our shared vision amongst several different provider teams has allowed our center to critically assess outcomes and continually revise our management strategies. Our patients have benefited from specific adjustments to our management strategy, including fetal intervention when technically feasible, planned caesarean section delivery with emergency postnatal peratrial atrial septal balloon dilation/stenting, and PA banding with the assistance of a RADI flow wire. However, despite focused efforts on various high-risk single-ventricle patients, a recent 2:1 matched cohort of patients undergoing a traditional Norwood procedure versus initial HS1P demonstrated improved short-term survival in the early hazard phase with no difference in survival at 1 year, reinforcing that the severity of comorbid lesions may still be driving longterm outcomes despite the strategy selected. ${ }^{10}$ Specific to 
HLHS/IAS, our protocol has been utilized in 15 patients with outcomes that mirror those of other institutions (Figure 5). A robust analysis of morbidity and neurodevelopmental outcomes is essential to further assess potential superiority of our current strategy.

\section{CONCLUSIONS}

We believe the most valuable investment a center can make toward improving its outcomes in high-risk single-ventricle palliation is to create a programmatic, streamlined, team-based approach to managing complex neonatal cardiovascular lesions that allows for critical analyses of the processes in place. Improved outcomes for high-risk neonates with single-ventricle anatomy remains challenging. Systematic, team-based approaches will foster the ability to critically analyze current practices and make informed modifications as needed to optimize the best chance for survival and improved quality of life.

\section{Conflict of Interest Statement}

The authors reported no conflicts of interest.

The Journal policy requires editors and reviewers to disclose conflicts of interest and to decline handling or reviewing manuscripts for which they may have a conflict of interest. The editors and reviewers of this article have no conflicts of interest.

\section{References}

1. Rychik J, Rome J, Collins M, DeCampli W, Spray T. The hypoplastic left heart syndrome with intact atrial septum: atrial morphololgy, pulmonary vascular histopathology, and outcome. J Am Coll Cardiol. 1999; 34:554-60.

2. Glatz JA, Tabbut S, Gaynor JW, Rome JJ, Montenegro L, Spray TL, et al. Hypoplastic left heart syndrome with atrial level restriction in the era of prenatal diagnosis. Ann Thorac Surg. 2007;84:1633-8.

3. Vlahos P, Lock J, McElhinney D, Van der Velde M. Hypoplastic left heart syndrome with intact or highly restrictive atrial septum. Circulation. 2004;109: 2326-30.

4. Graziano J, Heidelberger K, Ensing G, Ludomirsky A. The influence of a restrictive atrial septal defect on pulmonary vascular morphology in patients with hypoplastic left heart syndrome. Pediatr Cardiol. 2002;23:146-51.

5. Marshall A, Van der Velde M, Tworetzky W, Gomez CA, Wilkins-Haug L, Benson CB, et al. Creation of an atrial septal defect in utero for fetuses with hypoplastic left heart syndrome and intact or highly restrictive atrial septum. Circulation. 2004;110:253-8.

6. Jantzen D, Moon-Grady A, Morris S, Armstrong A, Berg C, Dangel J, et al. Hypoplastic left heart syndrome with intact of restrictive atrial septum. Circulation. 2017; 136:1346-9.

7. Vida V, Bacha E, Larrazabal A, Gauvreau K, Thiagaragan R, Fynn-Thompson F, et al. Hypoplastic left heart syndrome with intact or highly restrictive atrial septum: surgical experience from a single center. Ann Thorac Surg. 2007;84: $581-5$.

8. Jenkins P, Flanagan M, Jenkins K. Survival analysis and risk factors for mortality in transplantation and staged surgery for hypoplastic left heart syndrome. J Am Coll Cardiol. 2000;36:1178-85.

9. Sower CT, Romano JC, Yu S, Lowery R, Pasquali SK, Zampi JD. Early and midterm outcomes in high-risk single ventricle patients: hybrid vs Norwood palliation. Ann Thorac Surg. 2019;108:1849-55.

10. Zampi J, Hirsch J, Goldstein B, Armstrong A. Use of a pressure guidewire to assess pulmonary artery band adequacy in the hybrid stage 1 procedure for high-risk neonates with hypoplastic left heart syndrome and variants. Congenit Heart Dis. 2013;8:149-58. 